\title{
Fatal Calf Pneumonia Outbreaks in Italian Dairy Herds Involving Mycoplasma bovis and Other Agents of BRD Complex
}

\begin{abstract}
Angela Fanelli ${ }^{1+}$, Margie Cirilli ${ }^{1}$, Maria Stella Lucente ${ }^{1}$, Aya Attia Koraney Zarea ${ }^{1,2}$, Domenico Buonavoglia ${ }^{1}$, Maria Tempesta ${ }^{1}$ and Grazia Greco ${ }^{1 * t}$
\end{abstract}

${ }^{1}$ Department of Veterinary Medicine, University of Bari Aldo Moro, Bari, Italy, ${ }^{2}$ Department of Microbiology and Immunology, National Research Centre, Cairo, Egypt

Mycoplasma bovis is increasingly recognized worldwide as an important cause of disease with major welfare and production impairments on cattle rearing. Although it was detected in veal calves and beef cattle, little is known on the infection impact and on its temporal morbidity pattern in Italian dairy herds. Thus, this study aimed to investigate the involvement of $M$. bovis on fatal calf pneumonia outbreaks that occurred during 2009-2019 in 64 Italian dairy farms. Furthermore, a deeper diagnostic workup of concurrent infection with other viral and bacterial respiratory pathogens was assessed. Out of the investigated fatal pneumonia cases, $M$. bovis was frequently detected (animal prevalence, 16.16\%; 95\% Cl, 11.82-21.33; herd prevalence, 26.56; $95 \% \mathrm{Cl}, 16.29-39.08)$ either as the single agent of the disease in more than half of the positive samples (20/37) or in concurrent infections with Histophilus somni (9/37, 24.3\%), Mannheimia haemolytica (6/37, 16.621\%), Trueperella pyogenes (1/37, 2.70\%), Pasteurella multocida $(1 / 37,2.70 \%)$, bovine respiratory syncytial virus $(5 / 37,13.51 \%)$, and bovine viral diarrhea virus (2/37, 5.55\%). Based on time-series analysis, M. bovis was recorded in the area since 2009 with outbreaks displaying a clear morbidity seasonal pattern with peaks in April (43.21\%) and in September (13.51\%). This might be due to the stressing conditions during spring and late summer periods. Results of this study highlight that $M$. bovis infection warrants consideration, and control measures are needed given its involvement in lethal pneumonia outbreaks in dairy herds from an extended area.

Keywords: Mycoplasma bovis, bovine respiratory disease (BRD), fatal pneumonia, seasonal-trend decomposition based on loess (STL), Italy

\section{INTRODUCTION}

Mycoplasma bovis is a cell-wall-less bacterial pathogen, included in the class of Mollicutes (1). It is recognized as a causative agent of several diseases in cattle that have severe economic consequences for producers (1). In dairy cattle, $M$. bovis is probably the most common causative agent of mycoplasma mastitis, with arthritis and otitis media sometimes observed in infected animals (2). Noteworthy, it contributes to the occurrence of the bovine respiratory disease complex (BRD), a multifactorial syndrome consisting of chronic bronchopneumonia and pharyngitis, although often the infection can remain subclinical (3). 
Under natural condition, M. bovis interanimal transmission occurs mainly via colostrum, milk, air-borne, intrauterine, and contaminated semen (4). Moreover, M. bovis has the capability to produce a biofilm, making it possible for the bacterium to survive in the environment while withstanding the action of heat or desiccation (5). It was shown that M. bovis is able to maintain viability in the environment for months at low temperatures and weeks at room temperature on a variety of substrates in infected herds (5).

After infection, $M$. bovis can spread through the bloodstream, establishing a long-term persistent infection through escaping the immune response. $M$. bovis, as primary agent or under the action of concomitant stressing factors such as weaning, transport, or relocation to feedlots, may impair the host immune system efficiency resulting in the onset of the disease (6) including severe, often fatal, pneumonia $(7,8)$. Case fatality is estimated to be $5-10 \%$ or higher in more severe cases, with morbidity reaching $35 \%$ (3).

Although the real economic burden of the disease has not yet been evaluated, in the last few years, M. bovis has been increasingly recognized as a significant concern for the cattle industry due to milk loss, decreased weight gain, and cost for veterinary and drug treatments. Furthermore, M. bovis infection is particularly important from an animal welfare perspective being the persistent debilitating disease that is unresponsive to therapy (9). Indeed, the antibiotic resistance, the presence of asymptomatic carriers, and the lack of effective vaccines are identified as the major constraints in the control of the disease (10).

M. bovis was included in the EU-funded DISCONTOOLS project (https://www.discontools.eu/), which provides a decision tool for diseases prioritization in order to improve the application of preventive and control measures (11). According to epidemiological investigations, the most effective way to prevent the infection spread is based on the strict adoption of biosecurity measures and restrictions on animal movements (12). Currently, although in Europe, $M$. bovis occurrence does not incur official restrictions on livestock trade, nevertheless, some importing countries are requesting cattle to test free from the infection (10).

In Italy, $M$. bovis was detected in veal calves and beef cattle with pneumonic lesions at slaughter (13) and in batches of imported bulls stabled in farms in northern Italy (14). The infection was also recently reported in healthy and BRD symptomatic beef cattle imported from France in Southern Italy (15). Nonetheless, information on $M$. bovis infection in dairy herds is scarce.

In this study, we document the involvement of $M$. bovis in fatal calf pneumonia outbreaks in Italian dairy herds together with its spatial-temporal distribution. Additionally, concurrent infections with other viral and bacterial respiratory pathogens were recorded.

\section{MATERIALS AND METHODS}

From 2009 to 2019, 229 lung samples of calves ( $<11$ months) from 64 dairy farms experiencing outbreaks of fatal calf pneumonia were submitted for postmortem diagnostic workup to the Laboratory of Infectious Diseases of the Department of Veterinary Medicine of the University of Bari (Italy). The farms were from Apulia, Basilicata, Campania, and Calabria regions and managed under either semi-intensive production systems farming, characterized by access to pasture grazing during certain periods of the year or intensive farming, in case of larger herds, where animals were housed in a free stabling system.

M. bovis and a panel of concurrent infectious agents involved in BRD were investigated. In detail, bacteria and viruses including Histophilus somni, Mannheimia haemolytica, Pasteurella multocida, Trueperella pyogenes, bovine herpesvirus1 (BoHV-1), bovine viral diarrhea virus (BVDV), bovine respiratory syncytial virus (BRSV), and bovine coronavirus (BCoV) were included. Samples were submitted for DNA and RNA extraction using commercial kits (Qiagen, Milan, Italy) and subsequently analyzed for the infectious agents by using quantitative PCR (qPCR) or conventional PCR (cPCR) assays as already described (Supplementary Table S1). For each pathogen, prevalence was computed at animal (AP) and herd (HP) levels along with the $95 \%$ confidence interval $(95 \% \mathrm{CI})$. The spatial distribution of farms that tested positive for M. bovis was mapped by using QGIS software version 3.6.0 (16). To respect farmers' privacy, herds were represented at municipality level. A heatmap was built to better visualize the case distribution. The analysis of pathogen species co-occurrence was performed to detect pairs of pathogens that infect hosts more or less frequently than expected. A probabilistic model, as developed by Veech (17), was used to test for pairwise patterns of species co-occurrence, with the significance level $\alpha$, set at $\leq 0.05$.

With the aim of providing valuable insights on disease patterns, time-series analysis was performed, which is being widely implemented in the field of epidemiology (18-21). For the purpose of this study, an outbreak was defined as one of more cases occurring in the same epidemiological unit and month. The number of $M$. bovis outbreaks by calendar month was formatted into time series, and a seasonal trend decomposition based on loess (STL) was used to identify relevant seasonal patterns. Records from 2019 were excluded in the temporal analysis, as data for some months was missing. To extract the seasonality, the loess window was assigned to 13 , as it is recommended to use the next odd number following the number of observations in each seasonal cycle (22). Scale bars were included in the plots to describe the range of each component of the decomposition. The relative interquartile range (IQR) was used to measure the variability in the data explained by each component. The relative IQR is computed as the IQR of each component of the decomposition compared to the IQR of the raw data. Quantile plot of the residuals was drawn to ensure that they approximate a normal distribution. All the statistics were done using $\mathrm{R}$ software $3.5 .2(23)$.

\section{RESULTS}

The prevalence values at animal (AP) and herd (HP) level for all the detected bacteria and viruses are displayed in Table 1. $M$. bovis was the most frequently detected pathogen in the study area (AP, 16.16\%; 95\%CI, 11.82-21.33; HP, 26.56\%; 95\%CI, $16.29 \%-39.08 \%$ ) (Figure 1). Furthermore, M. bovis was detected 
TABLE 1 | Prevalence at animal and herd levels for Mycoplasma bovis and other pathogens associated with fatal pneumonia in farms from Southern Italy.

\begin{tabular}{|c|c|c|}
\hline Pathogen & $\begin{array}{c}\text { Animal prevalence } \\
(\%)(95 \% \mathrm{Cl})\end{array}$ & $\begin{array}{l}\text { Herd prevalence }(\%) \\
(95 \% \mathrm{Cl})\end{array}$ \\
\hline M. bovis & $16.16(11.82-21.33)$ & 26.56 (16.29-39.08) \\
\hline P. multocida & $4.80(2.42-8.43)$ & $6.25(1.72-15.23)$ \\
\hline M. haemolytica & 13.53 (9.38-18.66) & $20.31(11.28-32.22)$ \\
\hline H. somni & 7.86 (4.72-12.13) & 7.81 (2.58-17.29) \\
\hline T. pyogenes & $1.31(0.03-3.78)$ & $3.12(0.38-10.87)$ \\
\hline $\mathrm{BoHV}-1$ & $4.80(2.42-8.43)$ & $12.50(5.55-23.15)$ \\
\hline BVDV & 9.17 (5.76-13.67) & $12.50(5.55-23.15)$ \\
\hline BRSV & $6.98(4.04-11.09)$ & 9.37 (3.51-19.29) \\
\hline $\mathrm{BCoV}$ & 11.79 (7.91-16.68) & 3.12 (0.38-10.83) \\
\hline
\end{tabular}

BoHV-1, bovine herpesvirus-1; BVDV, bovine viral diarrhea virus; BRSV, bovine respiratory syncytial virus; BCoV, bovine coronavirus.

as single pathogen in more than half of the positive lung samples $(20 / 37)$. Details of the coinfections $(n=17)$ are provided in Supplementary Table S2. In particular, $M$. bovis was detected with $P$. multocida $(n=1,2.70 \%), M$. haemolytica ( $n=6$, $16.21 \%), H$. somni $(n=9,24.3 \%)$, and $T$. pyogenes $(n=$ $1,2.70 \%)$, and BVDV $(n=2,5.54 \%)$, and BRSV $(n=5$, $13.51 \%)$. No mixed infection of M. bovis with BCoV or BoHV-1 was recorded.

Out of 36 potential species pairs (Figure 2), 23 were considered in the co-occurrence analysis after removing the other $13(36.11 \%)$ with the expected outcomes lower than one. The majority of the pathogen pairs had random associations, while non-random associations were recorded for a few pairs including $M$. bovis-H. somni, P. multocida-BVDV, BVDVBRSV, and BRSV-H. somini (Figure 2). Furthermore, a negative significant association was detected for the M. bovis and $\mathrm{BCoV}$ pair, as these two species co-occurred at a frequency less than expected.

From 2009 to 2018, 37 outbreaks associated with M. bovis infection were recorded, with the majority occurring in 2015 ( $n$ $=6)$ and $2018(n=5)$. Considering the STL decomposition, the scale bars included in the plots showed that the remainder component of the decomposition had the highest IQR (Figure 3). This is mainly due to the considerable peak of outbreaks observed in 2015 and 2018, for which trend and seasonality components were not able to explain the variability of the data. The relative IQR measures, which exclude extreme values, were $37.66 \%$ for the seasonal component, $19.23 \%$ for the trend component, and $40.59 \%$ for the remainder. The seasonality pattern was constant over the period of analysis. Out of $37 \mathrm{M}$. bovis outbreaks, 16 (43.21\%) occurred in April, 5 (13.51\%) in September, and 4 (10.81\%) in December, with only sporadic events spread over other months (i.e., January, March, October, or November) (Supplementary Figure S1).

\section{DISCUSSION}

In this study, $M$. bovis was the agent most frequently detected from fatal calf pneumonia cases (AP, 16.16\%; 95\%CI,
11.82-21.33) occurring in dairy farms (HP, 26.56; 95\%CI, 16.29-39.08) from Southern Italy during a 10 -year period. $M$. bovis was detected as the single agent in half of positive lungs or in association with $H$. somni $(9 / 37,24.3 \%)$, followed by M. haemolytica (6/37, 16.21\%), T. pyogenes (1/37, 2.70\%), P. multocida $(1 / 37,2.70 \%), \operatorname{BRSV}(5 / 37,13.51 \%)$, and BVDV $(2 / 37$, $5.54 \%$ ) (Figure 2). However, as the samples all came from fatal cases, it was not possible to determine how prevalent this pathogen is in non-fatal cases.

$M$. bovis occurrence has been described worldwide with an increasing number of reports of calf pneumonia cases from Britain (24), Ireland (25), Canada (26, 27), and Switzerland (28). In Italy, M. bovis, together with other BRD agents, was recently reported both in healthy and sick animals $(14,15,29)$, stressing the importance of the agent as a primary concern in the Italian feedlot system. To date, no studies investigated fatal pneumonia outbreaks involving $M$. bovis with regard their temporal pattern. Thus, this work provides an important contribution to the knowledge on $M$. bovis, representing the first report investigating a series of fatal calf pneumonia outbreaks that occurred in dairy herds during a 10-year period in Southern Italy.

It is well-known that BRD is a multifactorial syndrome involving different bacterial or viral infectious agents and management factors, thus posing a real challenge in the diagnose without laboratory investigations $(28,30)$. As reported in previous studies, $M$. bovis was found to be positively associated with BVDV infection (Figure 2), although at low frequency, thus supporting for the synergistic role of the two pathogens in the occurrence of fatal bronchopneumonia $(27,31)$. Furthermore, there was evidence for a significant association between cooccurrence of M. bovis and H. somni and fatal pneumonia cases. This finding is of particular interest, $H$. somni being an emerging pathogen in the study area (15). Furthermore, H. somni was also shown to be widely implicated in pneumonia cases of cattle under 12 months of age in Ireland (32). Finally, in line with previous studies, coinfection of $M$. bovis and M. haemolytica was found (33), although the probabilistic model analysis suggests that this was a random association.

The main objective of the study was to investigate the occurrence of $M$. bovis in fatal calf pneumonia cases. Nevertheless, this work also highlighted the spread of other viral and bacterial respiratory pathogens in Italian dairy herds. Indeed, in line with previous studies, significant associations between the pairs of pathogens, including BVDV-BRSV (34) and BRSV-H. somni (35), were recorded. These pairs occurred at a frequency higher than expected, hinting that different distinct synergism of pairwise pathogens may occur in BRD pathogenesis.

Based on STL analysis, the occurrence of the M. bovis outbreaks revealed a time-dependent behavior with peaks in April (43.21\%) and September (13.51\%) (Figure 3). This is of particular importance, as detecting the relevant risk periods provides useful information for disease preparedness. Nevertheless, the seasonal pattern described in this work did not overlap those ones reported in previous studies performed in indoor systems that recorded the highest incidence 


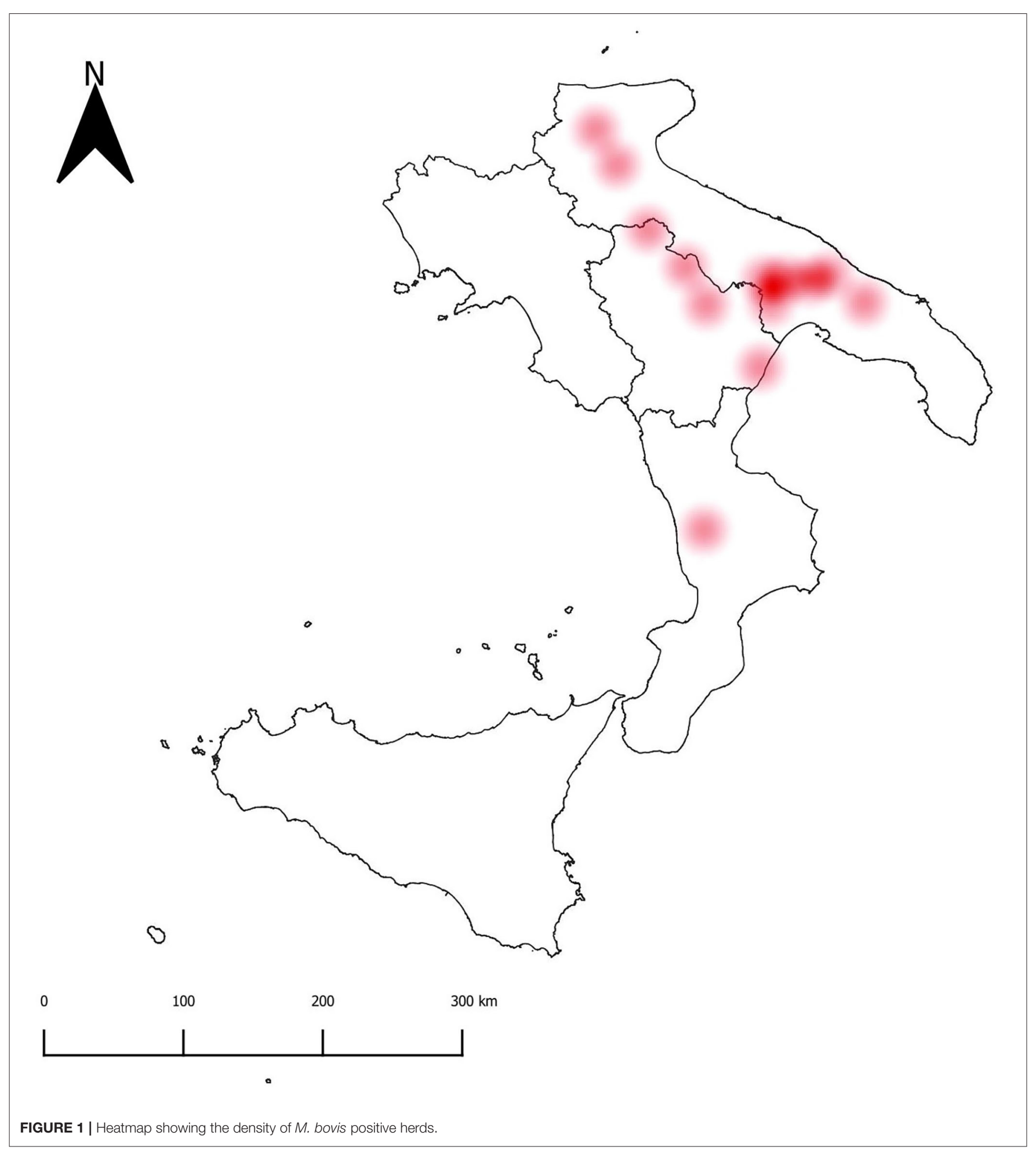

of pneumonia during colder seasons (14). Possibly, harsh temperature fluctuations rather than the cold temperatures may have played a role in the outbreak occurrence due to the remarkable changes in weather condition experienced during springtime and late summer in our study area. Given the seasonal importance, the effect of climatic stress on the hosts' disease susceptibility should be better investigated with further research.

Based on the time-series analysis, $M$. bovis occurrence was recorded in the area since 2009 (Figures 2, 3), but the sources 


\section{Species Co-occurrence Matrix}

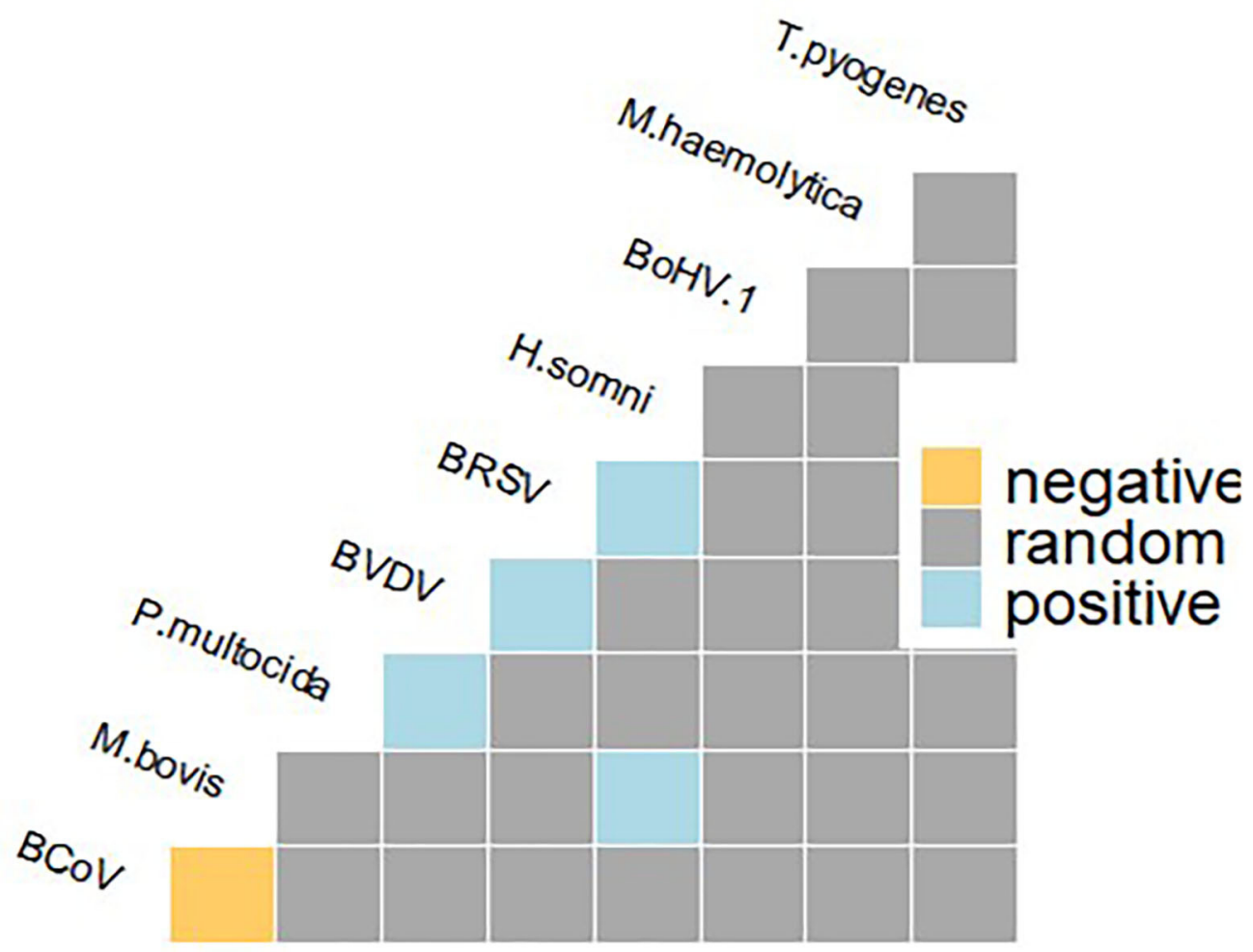

FIGURE 2 | Species co-occurrence matrix showing positive (in blue), negative (in yellow), and random (gray) associations.

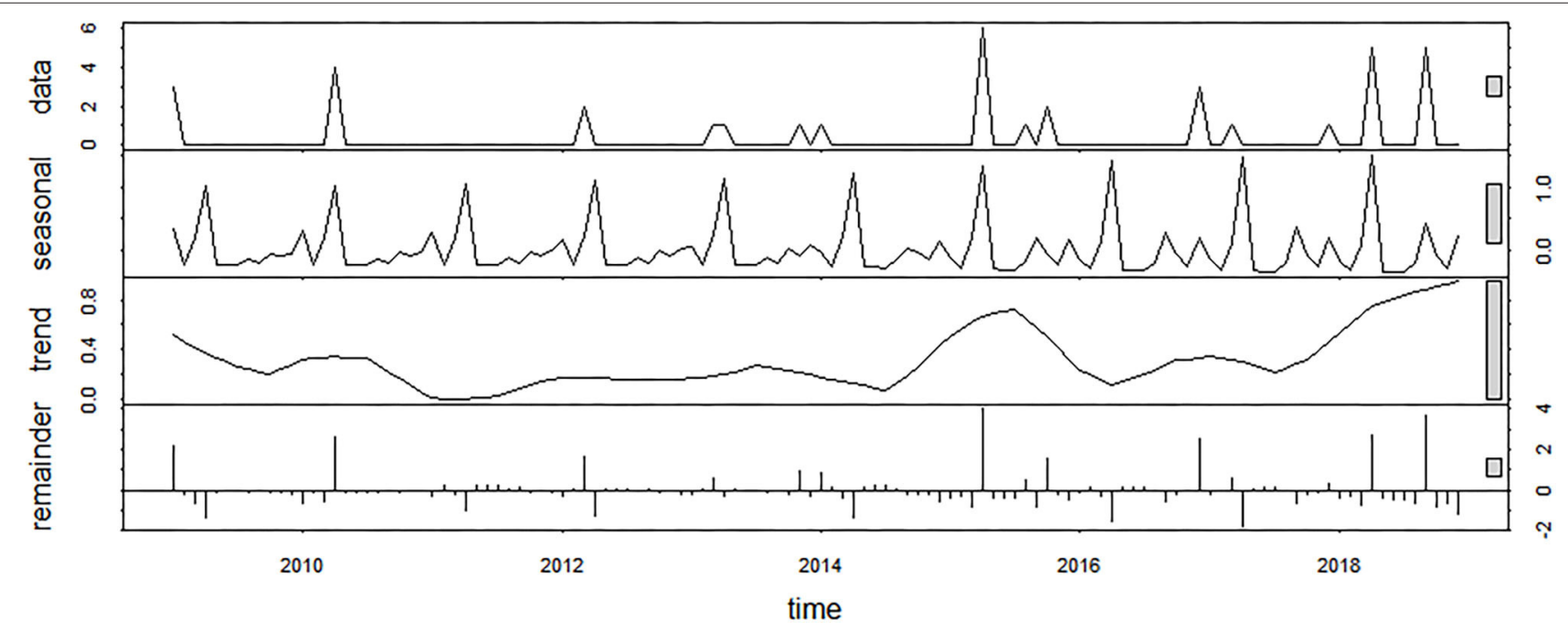

FIGURE 3 | Seasonal decomposition of $M$. bovis outbreaks using an additive model (raw data, seasonal component, trend component, and remainder).

for the outbreaks could not be identified, although the putative role of subclinical infected livestock introduced via the trade cannot be ruled out. Indeed, previous studies documented the introduction of the common BRD pathogens via imported cattle from France (15). On the other hand, other studies identified the presence of $M$. bovis infection in subclinical adult animals 
as the potential source of infection to the newly imported calves (14).

To conclude, based on the time-series analysis, $M$. bovis was in the area since 2009, with outbreaks displaying a clear morbidity seasonal pattern with peaks in April (43.21\%) and in September (13.51\%), which might be due to unknown stress conditions during spring and late summer periods. Results of this study highlight that M. bovis infection warrants consideration, and control measures are needed given its involvement in lethal pneumonia outbreaks in dairy herds from an extended area. One limitation of the present study is the lack of anamnestic data on the infected animals. Additionally, samples were not tested for bovine adenovirus (BAdV) and bovine parainfluenza virus 3 (BPIV-3), which have been recently found in BRD-affected beef steers in Southern Italy (36). Despite these limitations, results from this study made an important contribution from the animal health perspective.

\section{DATA AVAILABILITY STATEMENT}

The raw data supporting the conclusions of this article will be made available by the authors, without undue reservation.

\section{REFERENCES}

1. Brown DR, Whitcomb RF, Bradbury JM. Revised minimal standards for description of new species of the class Mollicutes (division Tenericutes). Int J Syst Evol Microbiol. (2007) 57:2703-19. doi: 10.1099/ijs.0.64722-0

2. Nicholas RAJ, Ayling RD. Mycoplasma bovis: disease, diagnosis, and control. Res Vet Sci. (2003) 74:105-12. doi: 10.1016/S0034-5288(02)00155-8

3. Nicholas R, Ayling R, McAuliffe L. Bovine respiratory disease. In: Nicholas R, Ayling R, McAuliffe L, editors. Mycoplasma Diseases of Ruminants. Oxfordshire: CABI Wallingford. p. 132-68. doi: 10.1079/9780851990125.0132

4. Dudek K, Nicholas RAJ, Szacawa E, Bednarek D. Mycoplasma bovis infections-occurrence, diagnosis and control. Pathogens. (2020) 9:121. doi: $10.3390 /$ pathogens 9080640

5. McAuliffe L, Ellis RJ, Miles K, Ayling. RD, Nicholas RAJ. Biofilm formation by mycoplasma species and its role in environmental persistence and survival. Microbiology. (2006) 152:913-22. doi: 10.1099/mic.0.2 8604-0

6. Perez-Casal J. Pathogenesis and virulence of Mycoplasma bovis. Vet Clin North Am Pract. (2020) 36:269-78. doi: 10.1016/j.cvfa.2020.02.002

7. Askar H, Chen S, Hao H, Yan X, Ma L, Liu Y, et al. Immune evasion of Mycoplasma bovis. Pathogens. (2021) 10:297. doi: 10.3390/pathogens100 30297

8. Khodakaram-Tafti A, Lopez A. Immunohistopathological findings in the lungs of calves naturally infected with Mycoplasma bovis. J Vet Med Ser A. (2004) 51:10-4. doi: 10.1111/j.1439-0442.2004.00596.x

9. Maunsell FP, Donovan GA. Mycoplasma bovis infections in young calves. Vet Clin North Am Food Anim Pract. (2009) 25:139-77. doi: 10.1016/j.cvfa.2008.10.011

10. Calcutt MJ, Lysnyansky I, Sachse K, Fox LK, Nicholas RAJ, Ayling RD. Gap analysis of Mycoplasma bovis disease, diagnosis and control: an aid to identify future development requirements. Transbound Emerg Dis. (2018) 65:91-109. doi: 10.1111/tbed.12860

11. Discontools. Diseases Databases. Available online at: https://www.discontools. eu/ (accessed April 14, 2021).

12. Nicholas RAJ, Fox LK, Lysnyansky I. Mycoplasma mastitis in cattle: to cull or not to cull. Vet J. (2016) 216:142-7. doi: 10.1016/j.tvjl.2016.08.001

13. Radaelli E, Luini M, Loria GR, Nicholas RAJ, Scanziani E. Bacteriological, serological, pathological and immunohistochemical studies of Mycoplasma

\section{ETHICS STATEMENT}

Ethical review and approval was not required for the animal study because The study included samples that were voluntary submitted from the animal owners to the diagnostic laboratory for diagnostic workup. Written informed consent was obtained from the owners for the participation of their animals in this study.

\section{AUTHOR CONTRIBUTIONS}

GG: conceptualization, data curation and analysis, methodology, investigation and supervision, and writing. AF: data curation, formal analysis, and writing-original draft preparation. $\mathrm{MC}$ and ML: laboratory analysis. AF, GG, MC, ML, AZ, DB, and MT: reviewing and editing. All authors contributed to the article and approved the submitted version.

\section{SUPPLEMENTARY MATERIAL}

The Supplementary Material for this article can be found online at: https://www.frontiersin.org/articles/10.3389/fvets. 2021.742785/full\#supplementary-material

bovis respiratory infection in veal calves and adult cattle at slaughter. Res Vet Sci. (2008) 85:282-90. doi: 10.1016/j.rvsc.2007.11.012

14. Catania S, Gastaldelli M, Schiavon E, Matucci A, Tondo A, Merenda $\mathrm{M}$, et al. Infection dynamics of mycoplasma bovis and other respiratory mycoplasmas in newly imported bulls on italian fattening farms. Pathogens. (2020) 9:537. doi: 10.3390/pathogens9070537

15. Pratelli A, Cirone F, Capozza P, Trotta A, Corrente M, Balestrieri A, et al. Bovine respiratory disease in beef calves supported long transport stress: an epidemiological study and strategies for control and prevention. Res Vet Sc. (2021) 135:450-5. doi: 10.1016/j.rvsc.2020.11.002

16. QGIS Development Team. QGIS Geographic Information System. Noosa: Open Source Geospatial Foundation (2019).

17. Veech JA. A probabilistic model for analysing species co-occurrence. Glob Ecol Biogeogr. (2013) 22:252-60. doi: 10.1111/j.1466-8238.2012.00789.x

18. Fanelli A, Tizzani P, Ferroglio E, Belleau E. Cheilospirura hamulosa in the Rock Partridge (Alectoris graeca saxatilis): epidemiological patterns and prediction of parasite distribution in France. Diversity. (2020) 12:484. doi: 10.3390/d12120484

19. Fanelli A, Tizzani P. Spatial and temporal analysis of varroosis from 2005 to 2018. Res Vet Sci. (2020) 131:215-21. doi: 10.1016/j.rvsc.2020.04.017

20. Iacopelli F, Fanelli A, Tizzani P, Berriatua E, Prieto P, Martínez-Carrasco $\mathrm{C}$, et al. Spatio-temporal patterns of sarcoptic mange in red deer and Iberian ibex in a multi-host natural park. Res Vet Sci. (2020) 128:2249. doi: 10.1016/j.rvsc.2019.11.014

21. Fanelli A, Perrone A, Ferroglio E. Spatial and temporal dynamics of wild boars Sus scrofa hunted in Alpine environment. Eur J Wildl Res. (2021) 67:47. doi: 10.1007/s10344-021-01484-4

22. Cleveland RB, Cleveland WS, McRae JE, Terpenning I. STL: a seasonaltrend decomposition procedure based on loess. J Off Stat. (1990) 6:3-37.

23. R Core Team. A Language and Environment for Statistical Computing. R Foundation for Statistical Computing (2018). Available online at: https:// www.r-project.org/ (accessed September 10, 2020).

24. Ayling RD, Bashiruddin SE, Nicholas RAJ. Mycoplasma species and related organisms isolated from ruminants in Britain between 1990 and 2000. Vet Rec. (2004) 155:413-6. doi: 10.1136/vr.155.14.413

25. Bell CJ, Blackburn P, Elliott M, Patterson TIAP, Ellison S, LahuertaMarin A, et al. Investigation of polymerase chain reaction assays to improve detection of bacterial involvement in bovine respiratory disease. 
J Vet Diagnostic Investig. (2014) 26:631-4. doi: 10.1177/104063871454 0166

26. Booker CW, Abutarbush SM, Morley PS, Jim GK, Pittman TJ, Schunicht OC, et al. Microbiological and histopathological findings in cases of fatal bovine respiratory disease of feedlot cattle in western Canada. Can Vet J. (2008) 49:473-81.

27. Gagea MI, Bateman KG, Shanahan RA, Van Dreumel T, McEwen BJ, Carman S, et al. Naturally occurring Mycoplasma bovis-associated pneumonia and polyarthritis in feedlot beef calves. J Vet Diagnostic Investig. (2006) 18:2940. doi: 10.1177/104063870601800105

28. Tschopp R, Bonnemain P, Nicolet J, Burnens A. Epidemiological study of risk factors for Mycoplasma bovis infections in fattening calves. Schweiz Arch Tierheilkd. (2001) 143:461-7.

29. Cirone F, Padalino B, Tullio D, Capozza P, Losurdo M, Lanave G, et al. Prevalence of pathogens related to bovine respiratory disease before and after transportation in beef steers: preliminary results. Animals. (2019) 9:17. doi: 10.3390/ani9121093

30. Szeredi L, Jánosi S, Pálfi V. Microbiological and pathological examination of fatal calf pneumonia cases induced by bacterial and viral respiratory pathogens. Acta Vet Hung. (2010) 58:341-56. doi: 10.1556/avet.58.2010.3.7

31. Haines DM, Martin KM, Clark EG, Kee Jim G, Janzen ED. The immunohistochemical detection of Mycoplasma bovis and bovine viral diarrhea virus in tissues of feedlot cattle with chronic, unresponsive respiratory disease and/or arthritis. Can Vet J. (2001) 42:857-60.

32. Murray GM, More SJ, Sammin D, Casey MJ, McElroy MC, O’Neill RG, et al. Pathogens, patterns of pneumonia, and epidemiologic risk factors associated with respiratory disease in recently weaned cattle in Ireland. J Vet Diagnostic Investig. (2017) 29:20-34. doi: 10.1177/1040638716674757

33. Caswell JL, Bateman KG, Cai HY, Castillo-Alcala F. Mycoplasma bovis in respiratory disease of feedlot cattle. Vet Clin North Am Food Anim Pract. (2010) 26:365-79. doi: 10.1016/j.cvfa.2010.03.003
34. Liu L, Hägglund S, Hakhverdyan M, Alenius S, Larsen LE, Belák S. Molecular epidemiology of bovine coronavirus on the basis of comparative analyses of the S gene. J Clin Microbiol. (2006) 44:95760. doi: 10.1128/JCM.44.3.957-960.2006

35. Corbeil LB. Histophilus somni host-parasite relationships. Anim Heal Res Rev. (2007) 8:151-60. doi: 10.1017/S146625230700 1417

36. Padalino B, Cirone F, Zappaterra M, Tullio D, Ficco G, Giustino A, et al. Factors affecting the development of bovine respiratory disease: a cross-sectional study in beef steers shipped from France to Italy. Front Vet Sci. (2021) 8:672. doi: 10.3389/fvets.2021.62 7894

Conflict of Interest: The authors declare that the research was conducted in the absence of any commercial or financial relationships that could be construed as a potential conflict of interest.

Publisher's Note: All claims expressed in this article are solely those of the authors and do not necessarily represent those of their affiliated organizations, or those of the publisher, the editors and the reviewers. Any product that may be evaluated in this article, or claim that may be made by its manufacturer, is not guaranteed or endorsed by the publisher.

Copyright (C) 2021 Fanelli, Cirilli, Lucente, Zarea, Buonavoglia, Tempesta and Greco. This is an open-access article distributed under the terms of the Creative Commons Attribution License (CC BY). The use, distribution or reproduction in other forums is permitted, provided the original author(s) and the copyright owner(s) are credited and that the original publication in this journal is cited, in accordance with accepted academic practice. No use, distribution or reproduction is permitted which does not comply with these terms. 